1 Hacettepe Journal of Mathematics and Statistics

$\bigcap$ Volume 45 (4) (2016), $1049-1060$

\title{
Application of modified optimal homotopy perturbation method to higher order boundary value problems in a finite domain
}

\author{
Fazal Ghani*, Saeed Islam ${ }^{\dagger}$, Cenap Ozel, ${ }^{\ddagger}$ Liaqat Ali§, M. M. Rashidi ฯ, \\ Tayebeh Hajjari ॥
}

\begin{abstract}
This research focuses on the solution of higher order boundary value problems by our proposed method "Modified Optimal Homotopy Perturbation Method" (MOHPM). A homotopy with an embedding parameter and Daftardar-Jafari polynomials are used. To control the convergence of solution, some auxiliary functions which depend upon variables and some constants are used. The proposed method is simple, rapid, effective and accurate. The accuracy has been proved by comparing our results with the solutions of optimal homotopy perturbation method (OHPM), optimal homotopy asymptotic method (OHAM), variational iterative method (VIM), variational iteration method using He's polynomials (VIMHP), homotopy perturbation method (HPM), Adoman decomposition method (ADM) and Quintic B-Spline Galerkin's scheme.
\end{abstract}

Keywords: Boundary value problems, Modified Optimal Homotopy Perturbation Method, Daftardar-Jafari Polynomials

Received : 19.02.2015 Accepted : 27.07.2015 Doi : 10.15672/HJMS.20164513109

*Abdul Wali Khan University Mardan, pakistan

${ }^{\dagger}$ Department of mathematics, Abdull Wali Khan University, Mardan, Pakistan.

$\ddagger$ Department of Mathematics, Dokuz Eylul University, Buca/Izmir,35160, Turkey, and KAU King Abdulaziz University Jeddah 21589 S. Arabia Email: cenap.ozel@gmail.com

$\S$ Abdul Wali Khan University Mardan, Pakistan \& Department of Electrical Engineering, CECOS University Peshawer KPK, Pakistan

IShanghai Automotive Wind Tunnel Center, Tongji Clean Energy Institute of advanced Study, China

"Department of Mathematics, Firoozkooh Branch, Islamic Azad University, Firoozkooh, Iran 


\section{INTRODUCTION}

Nonlinear boundary value problems have a significant contribution in today's modern fields of science and technology. They take place from steady state solutions of transient problems. The significance of higher order boundary value problems (BVPS) can be judge from their extensive use in mathematical modeling of different entities such as viscoelastic flows, hydrodynamic stability problems, non-Newtonian fluids and convection of heat etc [1]. In general, an nth order BVP can be represented as:

$$
w^{(p)}=\xi\left(w, w^{\prime}, \ldots w^{(p-1)}\right)+\Theta(s), \quad f<s<h
$$

having boundary conditions

$$
w^{(q)}(f)=\eta_{i} \text { and } w^{(q)}(h)=\lambda_{i},
$$

where $(q<p)$ is a non-negative integer, $\eta_{i}$ and $\lambda_{i}$ are real finite constants and $\Theta(s)$ is a continuous function on $[f, h]$.

Finding a solution for the above type differential equations is a tedious job. One may find an exact solution, but if the degree of non linearity is high, it becomes impossible to get an exact solution. Researchers have therefore focused on analytic solutions of such type problems. In literature we come across different analytic methods. Some of the interesting analytic methods which can be applied to a wide range of high order differential equations are homotopy analysis method (HAM) [2-4], homotopy perturbation method (HPM) [5-6], Adomian decomposition method (ADM) [7], optimal homotopy asymptotic method (OHAM) [8-12], optimal homotopy perturbation method (OHPM) [13-15] and variational iteration method (VIM) [16-18]. In order to obtain best approximate solution of differential equations, researchers in the field modify the existing analytical methods time to time. One such modification of (HPM) has been done by V. Marinca et al [13-15].The basis for their new method, which is known as (OHPM) is He's homotopy perturbation method. This method is developed on the same lines as was done earlier in He's homotopy perturbation method. A visible change in (OHPM) is that the non linear function is extended in series form for the parameter involved and auxiliary functions are inducted within the coefficients of this truncated series. In (OHPM) the auxiliary functions have unknown parameters which can be determined optimally. All these techniques give (OHPM) an edge over the conventional (HPM).

Our purpose in this paper is to obtain a new version of OHPM, which produces more accurate and reliable results than OHPM. The target is achieved here by introducing Daftardar-Jafferi polynomials in OHPM. The modified version of OHPM thus obtained will have its name as modified optimal homotopy perturbation method (MOHPM). It is important to note that these polynomials were defined in Daftatdar-Jafferi Method (DJM)[19], and basically are the non linear terms of the Taylor's series. S. Bhalekar et al. [20] have proved the convergence of these polynomials. It can be observed from the solved problems in section 3 that MOHPM is a powerful method as it converts a complex problem into a simpler one, which can then be solved easily. This method has great potential to solve ordinary differential equations of any order. The same technique can also be extended to solve partial differential equations, Integro-differential equations and system of differential equations of physical phenomenon. In our coming papers we will be showing the application of MOHPM to these types of problems. Here we have solved some linear and non linear higher order BVPS by MOHPM and OHPM to confirm the difference in obtained solutions. The results of MOHPM are also compared with those of exact solutions and the errors are compared with the already existing well-known results of OHAM, VIM, HPM, VIMHP, ADM and B-Spline. Numerical results show that MOHPM is found best in giving better and more accurate results. 
This manuscript is arranged as follows: In part 2, introduction of MOHPM is given. Part 3 is devoted to the application of MOHPM to higher order BVPS. In the same section results of numerical simulation using Mathematica 7.0 are given. In next and final section a concluding remarks are given for the obtained results.

\section{INTRODUCTION OF MOHPM}

Consider the problem

$$
\begin{aligned}
& \xi(\kappa(s))+\zeta(\kappa(s))-\Gamma(s)=0, \quad s \in \Omega \\
& \Delta\left(\kappa, \frac{\partial \kappa}{\partial s}\right)=0, s \in \Pi,
\end{aligned}
$$

where $\xi$ and $\zeta$ are linear and nonlinear operators respectively, $\Delta$ is the boundary operator, $\Pi$ is the boundary of the domain $\Omega, \Gamma$ is the analytic function and the differential along the normal drawn outwards from $\Omega$ is represented by $\frac{\partial}{\partial s}$. According to homotopy scheme, we create a homotopy, $\tilde{\kappa}(s, \vartheta): \Omega \times[0,1] \rightarrow R$ by

$$
\begin{aligned}
\Sigma(\tilde{\kappa}, \vartheta)= & (1-\vartheta)\left(\xi(\tilde{\kappa}(s, \vartheta))-\xi\left(\kappa_{i n i}(s, \vartheta)\right)\right) \\
& +\vartheta(\xi(\tilde{\kappa}(s, \vartheta))+\zeta(\tilde{\kappa}(s, \vartheta))-\Gamma(s))=0,
\end{aligned}
$$

where $\vartheta \in[0,1]$ is known as the embedding parameter and the initial guess for the solution of $(2.1)$ by $\kappa_{i n i}(s, \vartheta)$, which satisfies the boundary conditions. It is quite easy to note that, when $\vartheta=0$ and $\vartheta=1$ equation (2.3) holds and takes the form respectively as

$$
\begin{aligned}
& \Sigma(\tilde{\kappa}, 0)=\xi(\tilde{\kappa}(s, 0))-\xi\left(\kappa_{i n i}(s, 0)\right)=0, \\
& \Sigma(\tilde{\kappa}, 1)=\xi(\tilde{\kappa}(s, 1))+\zeta(\tilde{\kappa}(s, 1))-\Gamma(s)=0,
\end{aligned}
$$

thus change in $\vartheta$ from zero to one, will change the trivial solution for (2.4) to the solution of (2.5) continuously. That is, if $\vartheta$ changes from zero to one then $\tilde{\kappa}$ changes from $\kappa_{\text {ini }}$. to $\kappa$, this is known as deformation in topology. The paths $\xi(\tilde{\kappa}(s, 0))-\xi\left(\kappa_{i n i}(s, 0)\right)$ and $\xi(\tilde{\kappa}(s, 1))+\zeta(\tilde{\kappa}(s, 1))-\Gamma(s)$ are homotopic to each other. At this stage assume the perturbation series

$$
\tilde{\kappa}(s)=\tilde{\kappa}_{0}(s)+\vartheta \tilde{\kappa}_{1}(s)+\vartheta^{2} \tilde{\kappa}_{2}+\cdots .
$$

For MOHPM, the nonlinear function $\zeta(\tilde{\mu}(r, \theta))$ decomposes as

$$
\begin{aligned}
& \zeta(\tilde{\kappa}(s, \vartheta))=\zeta\left(\tilde{\kappa}_{0}(s)\right)+\vartheta\left(\zeta\left(\tilde{\kappa}_{0}(s)+\tilde{\kappa}_{1}(s)\right)-\zeta\left(\tilde{\kappa}_{0}(s)\right)\right) \\
& +\vartheta^{2}\left(\zeta\left(\tilde{\kappa}_{0}(s)+\tilde{\kappa}_{1}(s)+\tilde{\kappa}_{2}(s)\right)-\zeta\left(\tilde{\kappa}_{0}(s)+\tilde{\kappa}_{1}(s)\right)+\cdots\right.
\end{aligned}
$$

The terms $\zeta\left(\tilde{\kappa}_{0}(s)\right),\left\{\zeta\left(\tilde{\kappa}_{0}(s)+\tilde{\kappa}_{1}(s)\right)-\zeta\left(\tilde{\kappa}_{0}(s)\right)\right\},\left\{\zeta\left(\tilde{\kappa}_{0}(s)+\tilde{\kappa}_{1}(s)+\tilde{\kappa}_{2}(s)\right)-\zeta\left(\tilde{\kappa}_{0}(s)+\right.\right.$ $\left.\left.\tilde{\kappa}_{1}(s)\right)\right\}$ and so on, appearing in equation (2.7) on the right hand side are Daftardar-Jafari polynomials defined in [19]. Equation (2.7) can be written in a more compact form if we write $\zeta_{0}=\zeta\left(\tilde{\kappa}_{0}(s)\right)$ and $\zeta_{m}=\zeta\left(\sum_{i=0}^{m} \tilde{\kappa}_{i}(s)\right)-\zeta\left(\sum_{i=0}^{m-1} \tilde{\kappa}_{i}(s)\right)$. Thus, the expression (2.7) reduces to

$$
\zeta(\tilde{\kappa}(s, \vartheta))=\zeta_{0}+\sum_{j=1}^{\infty} \vartheta^{j} \zeta_{j} .
$$

putting back, equation (2.8) for equation (2.3), also by introducing a number of unknown auxiliary functions, $\varepsilon_{i}\left(s, c_{i}\right)$; for $i=0,1,2,3, \ldots$ that depend on the variable $\mathrm{s}$ and some constants $c_{0}, c_{1}, c_{2}, \ldots$, we get a new homotopy for $(2.1)$ as:

$$
\begin{aligned}
& \sum(\tilde{\kappa}, \vartheta)=(1-\vartheta)\left[\xi(\tilde{\kappa}(s, \vartheta))-\xi\left(\kappa_{i n i}(s, \vartheta)\right)\right] \\
& +\vartheta\left[\xi\left(\tilde{\kappa}(s, \vartheta)+\varepsilon_{0}\left(s, c_{0}\right) \zeta_{0}+\sum_{k=1}^{\infty} \varepsilon_{k}\left(s, c_{k}\right) \vartheta^{k} \zeta_{k}-\Gamma(s)\right]=0,\right.
\end{aligned}
$$


along with the boundary conditions:

$$
\Delta\left(\tilde{\kappa}(s, \vartheta), \frac{\partial}{\partial s}(\tilde{\kappa}(s, \vartheta))\right)=0 .
$$

Now, comparing the coefficients of similar powers of $\vartheta$ in (2.9), we get linear differential equations of zeroth order, first order, second order and so on, which can be solved very easily.

\section{Zeroth order problem:}

$$
\xi\left(\tilde{\kappa}_{0}(s)\right)=\xi\left(\kappa_{\text {ini }}(s)\right), \quad \Delta\left(\tilde{\kappa}_{0}, \frac{d \tilde{\kappa}_{0}}{d s}\right)=0
$$

\section{First order problem:}

$$
\xi\left(\tilde{\kappa}_{1}(s)\right)+\varepsilon_{0}\left(s, c_{o}\right) \zeta_{0}-\Gamma(s)=0, \quad \Delta\left(\tilde{\kappa}_{1}, \frac{d \tilde{\kappa}_{1}}{d s}\right)=0 .
$$

\section{Second order problem:}

$$
\xi\left(\tilde{\kappa}_{2}(s)\right)+\varepsilon_{1}\left(s, c_{1}\right)\left(\zeta_{1}\right)=0, \quad \Delta\left(\tilde{\kappa}_{2}, \frac{d \tilde{\kappa}_{2}}{d s}\right)=0 .
$$

\section{Third order problem:}

$$
\xi\left(\tilde{\kappa}_{3}(s)\right)+\varepsilon_{2}\left(s, c_{2}\right)\left(\zeta_{2}\right)=0, \quad \Delta\left(\tilde{\kappa}_{3}, \frac{d \tilde{\kappa}_{3}}{d s}\right)=0
$$

and so on

Where $\varepsilon_{i}\left(s, c_{i}\right) ; i=0,1,2,3, \ldots$, are auxiliary functions. The parameters $c_{i}$ 's are used to control the convergence and can itself be determined optimally. This can be done over the domain of the problem by minimizing the residual functional. In order to get an accurate result, solutions up to the higher order problems can be made but a solution up to third order will be sufficient. For $\vartheta=1$, if the series (2.7) converges, then the approximate solution is given by

$$
\kappa(s)=\tilde{\kappa}(s)=\tilde{\kappa}_{0}(s)+\tilde{\kappa}_{1}\left(s, c_{0}\right)+\tilde{\kappa}_{2}\left(s, c_{0}, c_{1}\right)+\tilde{\kappa}_{3}\left(s, c_{0}, c_{1}, c_{2}\right)+\cdots .
$$

The resulting residual can be obtained by backward substitution of equation (2.13) into equation (2.1) as

$$
\bar{R}\left(s, c_{0}, c_{1}, c_{2}, \ldots\right)=\xi(\tilde{\kappa}(s))+\zeta(\tilde{\kappa}(s))-\Gamma(s) .
$$

The exact solution $\tilde{\kappa}$, will be obtained if $\bar{R}=0$. In most of the problems usually $\bar{R} \neq 0$, and a minimization is needed over the domain of the problem. This can be done by using either least square's method, Galerkin's method or collocation method. When applying the method of least squares, we first introduce the functional

$$
\psi\left(c_{0}, c_{1}, c_{2}, \ldots\right)=\int_{t}^{h} \bar{R}^{2} d x
$$

and then minimizing it, we obtain

$$
\frac{\partial \psi}{\partial c_{0}}=\frac{\partial \psi}{\partial c_{1}}=\frac{\partial \psi}{\partial c_{2}}=\cdots=0 .
$$

For auxiliary constants we have to solve the following system, when applying Galerkin's method:

$$
\int_{t}^{h} \bar{R} \frac{\partial \tilde{\kappa}}{\partial c_{0}} d s=0, \quad \int_{t}^{h} \bar{R} \frac{\partial \tilde{\kappa}}{\partial c_{1}} d s=0, \quad \int_{t}^{h} \bar{R} \frac{\partial \tilde{\kappa}}{\partial c_{2}} d s=0, \ldots
$$




\section{APPLICATION OF OHPM AND MOHPM}

In this section high accuracy of MOHPM is shown over the existing methods in the literature. The proposed method is applied to some linear and non linear differential equations of different orders. As a result, we see that MOHPM gives best approximation and takes very less time to produce the solution.

Problem 1. Consider fifth order linear boundary value problem [10].

$$
\begin{aligned}
& \frac{d^{5} u}{d s^{5}}-u+15 e^{s}+10 \mathrm{~s} e^{s}=0, \quad 0<s<1, \\
& u(0)=0, u(1)=0, \quad u^{\prime}(0)=1, \quad u^{\prime}(1)=-e, u^{\prime \prime}(0)=0 .
\end{aligned}
$$

The exact solution for this problem is $u(s)=s(1-s) e^{s}$.

To apply MOHPM, we take:

$$
\begin{gathered}
u(s, \vartheta)=u_{0}(s)+\vartheta u_{1}(s)+\vartheta^{2} u_{2}(s), \\
\xi(\tilde{\kappa}(s, \vartheta))=\frac{d^{5} u(s, \vartheta)}{d s^{5}}, \xi\left(\kappa_{i n i}(s, \vartheta)\right)=0, \varepsilon_{0}\left(s, c_{0}\right)=1, \varepsilon_{1}\left(s, c_{1}\right)=1, \\
\xi(\tilde{\kappa}(s, \vartheta))+\zeta(\tilde{\kappa}(s, \vartheta))-\Gamma(s)=\frac{d^{5} u}{d s^{5}}-u+15 e^{s}+10 \mathrm{~s} e^{s} .
\end{gathered}
$$

Now put the above values in (2.9) and compare the coefficients of like powers of $\vartheta$ we get as:

Zeroth order problem:

$$
\left(u_{0}\right)^{(5)}(s)=0, u_{0}(0)=0, u_{0}(1)=0, u_{0}^{\prime}(0)=0, u_{0}^{\prime}(1)=-e, u_{0}^{\prime \prime}(0)=0 .
$$

First order problem:

$$
\begin{aligned}
& 15 \mathrm{e}^{s}+10 \mathrm{e}^{s} s-s u_{0}(s)+\left(u_{1}\right)^{(5)}(s)=0, \\
& u_{1}(0)=0, u_{1}(1)=0, u_{1}^{\prime}(0)=0, u_{1}^{\prime}(1)=0, u_{1}^{\prime \prime}(0)=0 .
\end{aligned}
$$

Second order problem:

$$
\begin{aligned}
& -s u_{1}(s)+\left(u_{2}\right)^{(5)}(s)=0, u_{2}(0)=0, u_{2}(1)=0, \\
& u_{2}^{\prime}(0)=0, u_{2}^{\prime}(1)=0, u_{2}^{\prime \prime}(0)=0 .
\end{aligned}
$$

Solve the above equations we obtain: $u_{0}(s), u_{1}(s), u_{2}(s)$, put these values in $(3.3)$ and also $\vartheta=1$, we get the following solution for $\mathrm{t}=0$ and $h=1$ :

$$
\begin{aligned}
& u(s)=s-0.500000000 s^{3}-0.333333333 s^{4}-0.125 s^{5} \\
& -0.033333333 s^{6}-0.0069444444 s^{7} \\
& -0.001190476 s^{8}-0.000173611 s^{9}-0.000022047 s^{10}-0.000002480 s^{11} \\
& -2.5052 \times 10^{-7} s^{12}-2.2967 \times 10^{-8} s^{13}-1.9261 \times 10^{-9} s^{14} .
\end{aligned}
$$

The results for problem 1 are shown in table-1 and figure- 1 as follows:

Problem 2. Fifth order non-linear boundary value problem [10].

$$
\begin{aligned}
& \frac{d^{5} u}{d s^{5}}-u^{2} e^{-s}=0, \quad 0<s<1, \\
& u(0)=1, \quad u^{\prime}(0)=1, u^{\prime \prime}(0)=1, u(1)=e, u^{\prime}(1)=e .
\end{aligned}
$$

Having exact solution $u(s)=e^{s}$. To solve this problem, we consider the second order approximation

$$
u(s)=u_{0}(s)+u_{1}\left(s, c_{0}\right)+u_{2}\left(s, c_{0}, c_{1}\right) .
$$


Let, $u(s, \vartheta)=u_{0}(s)+\vartheta u_{1}(s)+\vartheta^{2} u_{2}(s), \xi(\tilde{\kappa}(s, \vartheta))=\frac{d^{5} u(s, \vartheta)}{d s^{5}}, \xi\left(\kappa_{i n i}(s, \vartheta)\right)=0, \zeta(\tilde{\kappa}(s, \vartheta))=$ $u^{2}(s) e^{-s}, \varepsilon_{0}\left(s, c_{0}\right)=c_{0}, \varepsilon_{1}\left(s, c_{1}\right)=c_{1}, \varsigma_{0}=u_{0}^{2}(s), \varsigma_{1}=2 u_{0}(s) u_{1}(s)+u_{1}^{2}(s)$.

Put the above values in (2.9) and compare coefficients of like powers of $\vartheta$ we get as:

Zeroth order problem:

$$
\left(u_{0}\right)^{(5)}(s)=0, u_{0}(0)=1, u_{0}^{\prime}(0)=1, u_{0}^{\prime \prime}(0)=1, u_{0}(1)=e, u_{0}^{\prime}(1)=e .
$$

First order problem:

$$
\begin{aligned}
& -\mathrm{e}^{-s} c_{0} u_{0}(s)^{2}+\left(u_{1}\right)^{(5)}(s)=0, u_{1}(0)=0, \\
& u_{1}(1)=0, u_{1}^{\prime}(0)=0, u_{1}^{\prime}(1)=0, u_{1}^{\prime \prime}(0)=0 .
\end{aligned}
$$

\section{Second order problem:}

$$
\begin{aligned}
& -2 \mathrm{e}^{-s} c_{1} u_{0}(s) u_{1}(s)-\mathrm{e}^{-s} c_{1} u_{1}(s)^{2}+\left(u_{2}\right)^{(5)}(s)=0, \\
& u_{2}(0)=0, u_{2}(1)=0, u_{2}^{\prime}(0)=0, u_{2}^{\prime}(1)=0, u_{2}^{\prime \prime}(0)=0 .
\end{aligned}
$$

Solution of the above gives $u_{0}(s), u_{1}\left(s, c_{0}\right), u_{2}\left(s, c_{0}, c_{1}\right)$.

Now use (3.12) and apply the Galerkin's method consist of (2.15) and (2.18) we get the following values of $c_{i}^{\prime} s$ for $\mathrm{t}=0$ and $h=1$,

$$
c_{0}=0.999999240, \quad c_{1}=0.999758960 \text {. }
$$

The MOHPM approximate solution becomes:

$$
\begin{aligned}
& u(s)=1+s+\frac{s^{2}}{2}+0.166666667 s^{3}+0.041666667 s^{4}+\frac{s^{5}}{120}+\frac{s^{6}}{720}+\frac{s^{7}}{5040} \\
& \quad+0.000024802 s^{8}+0.000002756 s^{9}+2.7557 \times 10^{-7} s^{10}+2.5050 \times 10^{-8} s^{11} \\
& \quad+2.0928 \times 10^{-9} s^{12}+1.5506 \times 10^{-10} s^{13}+1.4690 \times 10^{-11} s^{14} .
\end{aligned}
$$

Now to check the accuracy of OHPM, we apply OHPM to (3.10) and obtain

$$
c_{0}=1.000553563, \quad c_{1}=-0.417820368 .
$$

The approximate solution by OHPM is then given as

$$
\begin{aligned}
& u(s)=1+s+\frac{s^{2}}{2}+0.166668432 s^{3}+0.041661159 s^{4}+0.008337946 s^{5} \\
& +0.00138968 s^{6}+0.000198523 s^{7}+0.000019825 s^{8}+0.000006842 s^{9} \\
& -5.0613 \times 10^{-7} s^{10}-4.1383 \times 10^{-8} s^{11}-1.6480 \times 10^{-8} s^{12} \\
& +1.4653 \times 10^{-8} s^{13}-9.8279 \times 10^{-9} s^{14}
\end{aligned}
$$

The results for problem 2 are shown in table-2 and figure-2 as follows:

Problem 3. Sixth order linear boundary value problem [17]

$$
\begin{aligned}
& \text { (3.18) } \frac{d^{6} u}{d s^{6}}-\left(u-6 e^{s}\right)=0, \quad 0<s<1, \\
& \text { (3.19) } u(0)=1, \mathrm{u}(1)=0, \mathrm{u}^{\prime \prime}(0)=-1, \mathrm{u}^{\prime \prime}(1)=-2 e, \mathrm{u}^{\prime \prime \prime \prime}(0)=-3, \mathrm{u}^{\prime \prime \prime \prime}(1)=-4 e .
\end{aligned}
$$

The exact solution for this problem is:

$$
u(s)=(1-s) e^{s} .
$$

To apply MOHPM, we use the steps used in problem-1 and in problem-2, we obtain approximate solution for $\mathrm{t}=0$ and $h=1$ as

$$
\begin{aligned}
& u(s)=1 .-3.5811 \times 10^{-12} s-0.5 s^{2}-0.333333333 s^{3}-0.125 s^{4}-0.0333333333 s^{5} \\
& -0.006944444 s^{6}-0.001190476 s^{7}-0.000173611 s^{8}-0.000022046 s^{9} \\
& -0.000002480 s^{10}-2.5052 \times 10^{-7} s^{11}-2.2964 \times 10^{-8} s^{12}
\end{aligned}
$$

The results for problem 3 are shown in table-3 and figure-3 as follows: 
(1) Table 3

(2) Figure 3

Problem 4. Sixth order nonlinear boundary value problem [24]

$$
\begin{aligned}
& \frac{d^{6} u}{d s^{6}}-u^{2} e^{s}=0, \quad 0<s<1, \\
& u(0)=1, u^{\prime}(0)=-1, u^{\prime \prime}(0)=1, u(1)=e^{-1}, u^{\prime}(1)-e^{-1}, u^{\prime \prime}(1)=e^{-1} .
\end{aligned}
$$

The exact solution is given as $u(s)=e^{-s}$.

To apply MOHPM, we consider the following second order approximation $u(s)=$ $u_{0}(s)+u_{1}\left(s, c_{0}\right)+u_{2}\left(s, c_{0}, c_{1}\right)$.

Now we use the steps mentioned in problem-1 and problem-2.

Using the Galerkin's method which consist of (2.15) and (2.18), we obtain the following values of $c_{i}^{\prime} s$ for $\mathrm{t}=0$ and $h=1$ :

$$
c_{0}=0.999781503, \quad c_{1}=0.568319310 .
$$

The approximate solution by MOHPM becomes:

$$
\begin{aligned}
u= & 1-s+\frac{s^{2}}{2}-0.1666666676 s^{3}+0.041666667 s^{4}-0.008333333 s^{5}+\frac{s^{6}}{720} \\
& -\frac{s^{7}}{5040}+\frac{s^{8}}{40320}-0.000002756 s^{9}+2.7556 \times 10^{-7} s^{10}-2.5048 \times 10^{-8} s^{11} \\
& +2.0862 \times 10^{-9} s^{12}-1.5896 \times 10^{-10} s^{13}+9.6037 \times 10^{-12} s^{14} .
\end{aligned}
$$

Also when we apply OHPM, we obtain the values of $c_{i}^{\prime} s$ as

$$
c_{0}=0.999986482, \quad c_{1}=0.991801518 \text {. }
$$

The approximate solution given by OHPM is

$$
\begin{aligned}
& u=1-s+\frac{s^{2}}{2}-0.166666667 s^{3}+0.041666667 s^{4}-0.008333334 s^{5} \\
& \quad+0.001388889 s^{6}-0.000198413 s^{7}+0.000024802 s^{8}-0.000002756 s^{9} \\
& \quad+2.7561 \times 10^{-7} s^{10}-2.5071 \times 10^{-8} s^{11}+2.0905 \times 10^{-9} s^{12} \\
& -1.5728 \times 10^{-10} s^{13}+7.1664 \times 10^{-12} s^{14}
\end{aligned}
$$

Results for problem 4 are given in table 4 and figure 4 as follows:

(1) Table 4

(2) Figure 4

\section{CONCLUSION}

In this paper a new idea has been developed and effectively applied to four higher order boundary value problems of fifth and sixth orders which provide very accurate results as compared to other well known methods in practice. Our proposed method has great potential to solve ordinary differential equations of any order. The same technique can also be extended to the solutions of partial differential equations, integro-differential equations and system of differential equations, the results obtained for these types of differential equations will be revealed in our coming papers. The merit of MOHPM is that it requires only a few terms to obtain accurate approximate solutions. This technique has a great robust, to attract engineer, scientists and researchers of every field. 


\section{References}

[1] S. Chandrasekhar, Hydrodynamic and hydro-magnetic stability, Dover Press, New York (1981).

[2] S. Liao, Beyond Perturbation: Introduction to the homotopy analysis method, Chapman \& Hall/CRC Press, Florida (2004).

[3] M.M. Rashidi, O. Anwar Beg, M.T. Rastegari, A Study of Non-Newtonian Flow and Heat Transfer over a Non-Isothermal Wedge using the Homotopy Analysis Method, Chemical Engineering Communications 199 (2012) 231-256.

[4] O. Anwar Beg, M.M. Rashidi, T.A. Beg, M. Asadi, Homotopy Analysis of Transient Magneto-Bio-Fluid Dynamics of Micropolar Squeeze Film in a Porous Medium: a Model for Magneto-Bio-Rheological Lubrication, Journal of Mechanics in Medicine and Biology, 12 (2012).

[5] H.J. He, Homotopy perturbation method for solving boundary value problems, Physics Letters A, 350 (2006) 87-88.

[6] M.M. Rashidi, D.D. Ganji, S. Dinarvand, Explicit Analytical Solutions of the Generalized Burger and Burger-Fisher Equations by Homotopy Perturbation Method, Numerical Methods for Partial Differential Equations, 25 (2009) 409-417.

[7] G. Adomian, A review of the decomposition method and some recent results for nonlinear equations, Computers and Mathematics with Applications, 21 (1991) 101-127.

[8] V. Marinca, N. Herisanu, An optimal homotopy asymptotic approach applied to non-linear MHD Jeffery-Hamel flow, Math. Probl. Eng., Art. N 169056, published 2011.

[9] V. Marinca, N. Herisanu andI. Nemes, Optimal homotopy asymptotic method for solving Blasius.equation, Appl. Math. Comput., 231 (2014) 134-139.

[10] J. Ali, S. Islam, S. Shah and H. Khan, The optimal homotopy asymptotic method for the solution of fifth and sixth order boundary value problems, World Applied Sciences Journal, 15 (2011) 1120-1126.

[11] N. Herisanu, V. Marinca, Gh.Madescu, An Analytical approach to non-linear dynamical model of a permanet magnet synchronous generator, Wind Energy, 2015,DOI:10.1002/we.1785

[12] N. Herisanu,V. Marinca, On the flow of a Walters-type B' viscoelastic fluid in a vertical channel with porous wall, Int.J.Heat and Mass Transfer, 79 (2014) 146-165.

[13] V. Marinca and N. Herisanu, Nonlinear dynamic analysis of an electrical machine rotorbearing system by optimal homotopy perturbation method, Computers and Mathematics with Applications, 61 (2011) 2019-2024.

[14] N. Herisanu and V. Marinca, Optimal homotopy perturbation method for non-conservative dynamical system of a rotating electrical machine, Zeitschrift fur Naturforschung A, 67.(2012) 509-516

[15] V. Marinca and N. Herisanu, Optimal homotopy perturbation approach to thin film flow of a fourth grade fluid, AIP Conference Proceedings, 1479 (2012) 2383-2386.

[16] M.A. Noor and S. T. Mohyud-Din,Variational iteration method for fifth order boundary value problems using He's polynomial, Mathematical Problems in Engineering, 10 (2008) ID 954794.

[17] M.A. Noor, K.I. Noor and S.T. Mohyud-Din,Variational iteration method for solving sixth order boundary value problems, communications in non linear science and numerical simulations, 14 (2009) 2571-2580.

[18] M.A. Noor and S.T. Mohyud-Din, Variational iteration technique for solving higher order boundary value problems, Applied Mathematics and Computation, 189 (2007) 1929-1942.

[19] V. Daftardar-Gejji and H. Jafari, An iterative method for solving nonlinear functional equations, Journal of Mathematical Analysis and Applications, 316 (2006) 753-763.

[20] S. Bhalekar and V. Daftardar-Gejji,Convergence of the New Iterative Method, International Journal of Differential Equations, 11 (2011) Article ID 989065,10pages

[21] J. Ali S. Islam,I. Ali and H. Khan, Application of optimal homotopy asymptotic method to higher order boundary value problems, Abstract and Applied Analysis, 12 Article ID 401217, 14 pages. 
Table 1. Table1 shows comparison of the errors obtained by (MOHPM)(3.9), (OHAM) [10], (VIMHP) [16], (VIM) [18], (B-Spline) [22], with the exact solution. We observe that the result of MOHPM is better and more accurate than the above mentioned methods.

\begin{tabular}{|c|c|c|c|c|c|c|c|}
\hline $\mathrm{S}$ & Exact & MOHPM & $\begin{array}{l}\mathrm{E}^{*} \\
(\mathrm{MOHPM}\end{array}$ & $\begin{array}{l}\mathrm{E}^{*} \\
\text { (OHAM) }\end{array}$ & $\begin{array}{l}\mathrm{E}^{*} \\
\text { (VIMHP) }\end{array}$ & $\begin{array}{l}\mathrm{E}^{*} \\
\text { (VIM) }\end{array}$ & $\begin{array}{l}\mathrm{E}^{*} \quad(\mathrm{~B} \\
\text { Spline) }\end{array}$ \\
\hline 0.0 & 0.000000 & 0.000000 & 0. & 0 . & 0 . & 0 . & 0 . \\
\hline 0.1 & 0.099465 & 0.099465 & $5.4 \times 10^{-14}$ & $9.0 \times 10^{-11}$ & $\begin{array}{l}- \\
3.0 \times 10^{-11}\end{array}$ & $1.0 \times 10^{-09}$ & $7.0 \times 10^{-04}$ \\
\hline 0.2 & 0.195424 & 0.195424 & $3.7 \times 10^{-13}$ & $4.0 \times 10^{-10}$ & $\begin{array}{l} \\
2.0 \times 10^{-10}\end{array}$ & $2.0 \times 10^{-09}$ & $\begin{array}{l}- \\
7.2 \times 10^{-04}\end{array}$ \\
\hline 0.3 & 0.283470 & 0.283470 & $1.0 \times 10^{-12}$ & $5.0 \times 10^{-10}$ & $4.0 \times 10^{-10}$ & $1.0 \times 10^{-09}$ & $4.1 \times 10^{-04}$ \\
\hline 0.4 & 0.358037 & 0.358037 & $1.9 \times 10^{-12}$ & $2.0 \times 10^{-11}$ & $\begin{array}{l}- \\
8.0 \times 10^{-10}\end{array}$ & $2.0 \times 10^{-09}$ & $4.6 \times 10^{-04}$ \\
\hline 0.5 & 0.412180 & 0.412180 & $2.7 \times 10^{-12}$ & $1.0 \times 10^{-09}$ & $\begin{array}{l}- \\
1.0 \times 10^{-09}\end{array}$ & $3.1 \times 10^{-08}$ & $4.7 \times 10^{-04}$ \\
\hline 0.6 & 0.43730 & 0.437308 & $3.0 \times 10^{-12}$ & $2.0 \times 10^{-09}$ & $\begin{array}{l}- \\
2.0 \times 10^{-09}\end{array}$ & $3.7 \times 10^{-08}$ & $4.8 \times 10^{-04}$ \\
\hline 0.7 & 0.422888 & 0.422888 & $2.1 \times 10^{-12}$ & $2.0 \times 10^{-09}$ & $\begin{array}{l}- \\
2.0 \times 10^{-09}\end{array}$ & $4.1 \times 10^{-08}$ & $3.9 \times 10^{-04}$ \\
\hline 0.8 & 0.356086 & 0.356086 & $3.7 \times 10^{-12}$ & $1.0 \times 10^{-09}$ & $2.0 \times 10^{-09}$ & $3.1 \times 10^{-08}$ & $3.1 \times 10^{-04}$ \\
\hline 0.9 & 0.221364 & 0.221364 & $\begin{array}{l}- \\
3.2 \times 10^{-11}\end{array}$ & $4.0 \times 10^{-10}$ & $\begin{array}{l}-1.0 \times 1- \\
-09\end{array}$ & $1.4 \times 10^{-08}$ & $1.6 \times 10^{-04}$ \\
\hline 1.0 & 0.000000 & $2.5 \times 10^{-8}$ & $-\overline{1.6 \times 10^{-10}}$ & 0. & 0. & 0. & 0. \\
\hline
\end{tabular}

[22] S.U. Islam and M.A. Khan, A numerical method based on polynomials sextic spline functions for the solution of special fifth-order boundary value problems, Applied Mathematics and Computation, 181 (2006) 356-361.

[23] A.M. Wazwaz, The numerical solution of sixth-order boundary value problems by the modified decomposition method, Applied Mathematics and Computation, 118 (2001) 311-325.

[24] M.A. Noor and S.T. Mohyud-Din, Homotopy perturbation method for solving sixth order boundary value problems, Computers and Mathematics with Applications, 55 (2008) 29532972 .

Table 1

$\mathrm{E}^{*}=$ Exact-Approx.

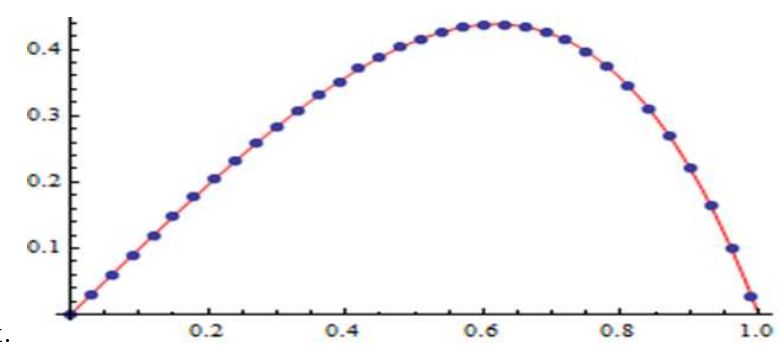

Figure 1: Dotted curve-sol: (MOHPM) and solid curve-sol: (Exact).

TABLE 2

$\mathrm{E}^{*}=$ Exact-Approx. 
Table 2. Table 2 shows comparison of the solutions obtained by MOHPM (3.16), OHPM (3.17), OHAM [10], VIM [18] and B-Spline [22]. From the numerical results it is clear that MOHPM is more efficient and more accurate.

\begin{tabular}{|c|c|c|c|c|c|c|c|}
\hline $\mathrm{S}$ & Exact & MOHPM & $\begin{array}{l}\text { E* } \\
\text { MOHPM }\end{array}$ & $\begin{array}{l}\mathrm{E}^{*} \\
\text { OHPM }\end{array}$ & $\begin{array}{l}\mathrm{E}^{*} \\
\text { OHAM }\end{array}$ & $\mathrm{E}^{*}$ VIM & $\begin{array}{l}\mathrm{E}^{*} \\
\text { B-Spline }\end{array}$ \\
\hline 0.0 & 1.000000 & 1.000000 & 0.0 & 0.0 & 0.0 & 0.0000 & 0.0000 \\
\hline 0.1 & 1.105170 & 1.105170 & $3.1 \times 10^{-15}$ & $1.2 \times 10^{-09}$ & $1.9 \times 10^{-10}$ & $3.0 \times 10^{-11}$ & ${ }^{-} 8.0 \times 10^{-03}$ \\
\hline 0.2 & 1.221402 & 1.221402 & $1.9 \times 10^{-14}$ & $6.8 \times 10^{-09}$ & $1.2 \times 10^{-09}$ & $-\overline{2.2 \times 10^{-10}}$ & $\begin{array}{l}- \\
1.2 \times 10^{-03}\end{array}$ \\
\hline 0.3 & 1.349858 & 1.349858 & $5.4 \times 10^{-14}$ & $1.4 \times 10^{-08}$ & $3.3 \times 10^{-09}$ & $-\overline{4} .0 \times 10^{-10}$ & $\begin{array}{l}- \\
5.0 \times 10^{-03}\end{array}$ \\
\hline 0.4 & 1.491824 & 1.491824 & $1.0 \times 10^{-13}$ & $2.0 \times 10^{-08}$ & $6.3 \times 10^{-09}$ & $8.0 \times 10^{-10}$ & $3.0 \times 10^{-03}$ \\
\hline 0.5 & 1.648721 & 1.648721 & $1.4 \times 10^{-13}$ & $2.1 \times 10^{-08}$ & $9.3 \times 10^{-09}$ & $\begin{array}{l}- \\
1.2 \times 10^{-09}\end{array}$ & $8.0 \times 10^{-03}$ \\
\hline 0.6 & 1.822118 & 1.822118 & $1.6 \times 10^{-13}$ & $1.7 \times 10^{-08}$ & $1.1 \times 10^{-08}$ & $-\overline{209 \times 10^{-09}}$ & $6.0 \times 10^{-03}$ \\
\hline 0.7 & 2.013752 & 2.013752 & $1.5 \times 10^{-13}$ & $1.2 \times 10^{-08}$ & $1.1 \times 10^{-08}$ & $\begin{array}{l}- \\
2.2 \times 10^{-09}\end{array}$ & -0.0000 \\
\hline 0.8 & 2.225540 & 2.225540 & $9.9 \times 10^{-14}$ & $7.0 \times 10^{-09}$ & $8.2 \times 10^{-09}$ & $1.9 \times 10^{-09}$ & $\begin{array}{l}9.0 \\
10^{-03}\end{array}$ \\
\hline 0.9 & 2.459603 & 2.459603 & $1.1 \times 10^{-14}$ & $2.0 \times 10^{-09}$ & $1.9 \times 10^{-09}$ & $-\overline{1.4 \times 10^{-09}}$ & $\begin{array}{l}- \\
9.0 \times 10^{-03}\end{array}$ \\
\hline 1.0 & 2.718281 & 2.718281 & $1.0 \mathrm{E}-13$ & 3.0E-09 & 0.00 & 0.000 & 0.0000 \\
\hline
\end{tabular}

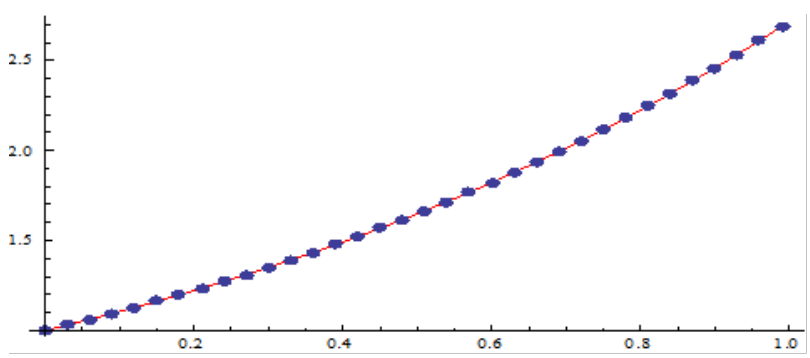

Figure 2: Dotted curve-sol: (MOHPM) and solid curve-sol: (Exact).

Table 3

$\mathrm{E}^{*}=$ Exact-Approx. 
Table 3. Table3shows comparison of the errors obtained by MOHPM( 3.20), OHAM [10], ADM [23], VIM [18] and HPM [24], with the exact solution. We observe that our results of MOHPM are better and more accurate than the above mentioned methods.

\begin{tabular}{|c|c|c|c|c|c|c|c|}
\hline $\mathrm{S}$ & Exact & MOHPM & $\begin{array}{l}\mathrm{E}^{*} \\
\text { MOHPM }\end{array}$ & $\begin{array}{l}\mathrm{E}^{*} \\
\text { OHAM }\end{array}$ & $\mathrm{E}^{*} \mathrm{ADM}$ & $\mathrm{E}^{*} \mathrm{VIM}$ & $\mathrm{E}^{*} \mathrm{HPM}$ \\
\hline 0.0 & 1. & 1. & 0. & 0. & 0 . & 0. & 0 . \\
\hline 0.1 & 0.994653 & 0.994653 & $3.5 \times 10^{-13}$ & $2.1 \times 10^{-08}$ & $4.1 \times 10^{-04}$ & $4.1 \times 10^{-04}$ & $4.1 \times 10^{-04}$ \\
\hline 0.2 & 0.977122 & 0.977122 & $6.7 \times 10^{-13}$ & $4.0 \times 10^{-08}$ & $7.8 \times 10^{-04}$ & $7.8 \times 10^{-04}$ & $7.8 \times 10^{-04}$ \\
\hline 0.3 & 0.944901 & 0.944901 & $9.2 \times 10^{-13}$ & $5.7 \times 10^{-08}$ & $1.1 \times 10^{-03}$ & $1.1 \times 10^{-03}$ & $-\overline{1.1 \times 10^{-03}}$ \\
\hline 0.4 & 0.895094 & 0.895094 & $1.1 \times 10^{-12}$ & $7.0 \times 10^{-08}$ & $-\overline{1.3 \times 10^{-03}}$ & $1.3 \times 10^{-03}$ & $-\overline{1.3 \times 10^{-03}}$ \\
\hline 0.5 & 0.824360 & 0.824360 & $9.0 \times 10^{-13}$ & $7.6 \times 10^{-08}$ & $\begin{array}{l}- \\
1.3 \times 10^{-03}\end{array}$ & $\begin{array}{l}- \\
1.3 \times 10^{-03}\end{array}$ & $\begin{array}{l}- \\
1.3 \times 10^{-03}\end{array}$ \\
\hline 0.6 & 0.728847 & 0.728847 & $\begin{array}{l}- \\
1.6 \times 10^{-12}\end{array}$ & $7.5 \times 10^{-08}$ & $-\overline{1.3 \times 10^{-03}}$ & $\begin{array}{l}- \\
1.3 \times 10^{-03}\end{array}$ & $\begin{array}{l}- \\
1.3 \times 10^{-03}\end{array}$ \\
\hline 0.7 & 0.604125 & 0.604125 & $-\overline{1.9 \times 10^{-11}}$ & $6.5 \times 10^{-08}$ & $-\overline{1.1 \times 10^{-03}}$ & $1.1 \times 10^{-03}$ & $-\overline{1.1 \times 10^{-03}}$ \\
\hline 0.8 & 0.445108 & 0.445108 & $1.1 \times 10^{-10}$ & $4.8 \times 10^{-08}$ & $4.1 \times 10^{-04}$ & $4.1 \times 10^{-04}$ & $4.1 \times 10^{-04}$ \\
\hline 0.9 & 0.245960 & 0.245960 & $\begin{array}{l}- \\
5.3 \times 10^{-10}\end{array}$ & $2.5 \times 10^{-08}$ & $\overline{-} 7.8 \times 10^{-04}$ & $7.8 \times 10^{-04}$ & $\begin{array}{l}- \\
7.8 \times 10^{-04}\end{array}$ \\
\hline 1.0 & 0. & $2.0 \mathrm{E}-\mathrm{O} 9$ & $\begin{array}{l}- \\
2.1 \times 10^{-09}\end{array}$ & $2.1 \times 10^{-09}$ & 0.0 & 0.0 & 0.0 \\
\hline
\end{tabular}

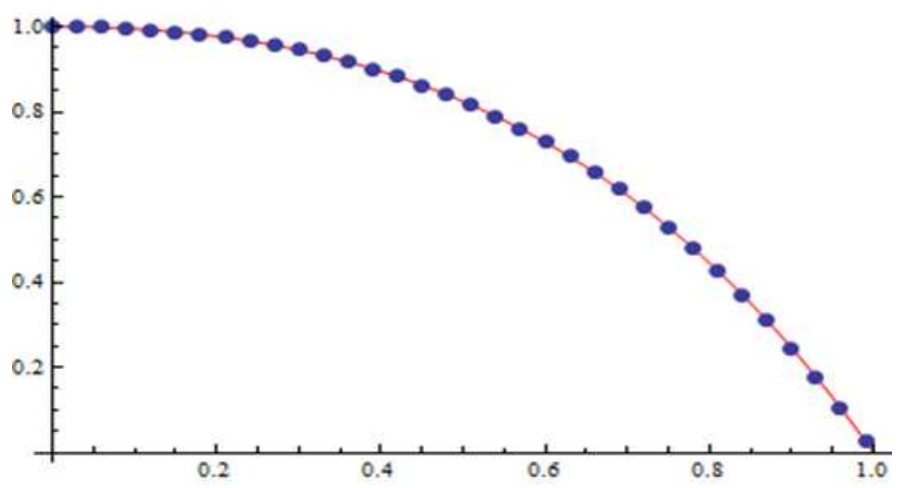

Figure 3: Dotted curve-sol: (MOHPM) and solid curve-sol: (Exact).

Table 4

$\mathrm{E}^{*}=$ Exact-Approx. 
Table 4. Table 4 shows comparison of errors obtained by MOHPM (3.23), OHPM (3.24), OHAM [21], ADM [23], VIM [18] and HPM [24], with the exact solution. Results indicate clearly that MOHPM gives better and accurate approximations than the above mentioned methods.

\begin{tabular}{|c|c|c|c|c|c|c|c|c|}
\hline $\mathrm{s}$ & Exact & MOHPM & $\begin{array}{l}\text { E* } \\
\text { MOHPM }\end{array}$ & $\begin{array}{l}\text { E* }^{*} \\
\text { OHPM }\end{array}$ & $\begin{array}{l}\text { E* } \\
\text { OHAM }\end{array}$ & $\begin{array}{l}E^{*} \\
\text { VIM }\end{array}$ & $\begin{array}{l}\mathrm{E}^{*} \\
\mathrm{ADM}\end{array}$ & $\begin{array}{l}\mathrm{E}^{*} \\
\mathrm{HPM}\end{array}$ \\
\hline 0.0 & 1.000000 & 1.000000 & 0.0 & 0.0 & 0 & 0 & 0 & 0 \\
\hline 0.1 & 0.904837 & 0.904837 & $4.1 \times 10$ & $1.2 \times 10^{-09}$ & $\begin{array}{l}-4.82 \\
\mathrm{x} 10^{-10}\end{array}$ & $2.3 \times 10^{-07}$ & $1.2 \times 10^{-04}$ & $1.2 \times 10^{-04}$ \\
\hline 0.2 & 0.818730 & 0.818730 & $2.4 \times 10^{-14}$ & $6.8 \times 10^{-09}$ & $4.92 \times 10^{-1}$ & $-\overline{1.3 \times 10^{-06}}$ & $\begin{array}{l}- \\
2.3 \times 10^{-04}\end{array}$ & $\begin{array}{l}- \\
2.3 \times 10^{-04}\end{array}$ \\
\hline 0.3 & 0.740818 & .740818 & $5 \times 10^{-14}$ & $1.4 \times 10^{-08}$ & $2.37 \times 10^{-1}$ & $3.3 \times 10^{-06}$ & $3.2 \times 10^{-04}$ & $\begin{array}{l} \\
.2 \times 10^{-04}\end{array}$ \\
\hline 0.4 & 0.670320 & 0.670320 & $3 \times 10^{-14}$ & $2.0 \times 10$ & $5.11 \times 10^{-1}$ & $5.2 \times 10^{-06}$ & $-\overline{3.8 \times 10^{-04}}$ & $-\overline{3.8 \times 10^{-04}}$ \\
\hline 0.5 & 0.606530 & 0.606530 & $9.3 \times 10^{-14}$ & $2.1 \times 10^{-08}$ & $6.42 \times 10^{-1}$ & $6.1 \times 10^{-06}$ & $4.0 \times 10^{-04}$ & $4.0 \times 10^{-04}$ \\
\hline 0.6 & 0.548811 & 0.548811 & $8.2 \times 10^{-14}$ & $1.7 \times 10^{-08}$ & $2.02 \times 10^{-1}$ & $5.7 \times 10^{-06}$ & $\begin{array}{l}- \\
3.9 \times 10^{-04}\end{array}$ & $3.9 \times 10^{-04}$ \\
\hline 0.7 & 0.496585 & 0.496585 & $5.5 \times 10^{-14}$ & $1.2 \times 10^{-08}$ & $\begin{array}{l}- \\
5.37 \times 10^{-1}\end{array}$ & $4.0 \times 10^{-06}$ & $3.3 \times 10^{-04}$ & $-\overline{3.3 \times 10^{-04}}$ \\
\hline 0.8 & 0.449328 & 0.449328 & $2.9 \times 10^{-14}$ & $7.0 \times 10^{-09}$ & $1.02 \times 10^{-0}$ & $1.9 \times 10^{-06}$ & $2.4 \times 10^{-04}$ & $\begin{array}{l}- \\
2.4 \times 10^{-04}\end{array}$ \\
\hline 0.9 & 0.406569 & 0.406569 & $3.1 \times 10^{-14}$ & $2.0 \times 10^{-09}$ & $8.23 \times 10^{-1}$ & $3.5 \times 10^{-07}$ & $-1.2 \times 10^{-04}$ & $-\overline{1.2 \times 10^{-04}}$ \\
\hline 1.0 & 0.367879 & 0.367879 & $1.1 \times 10^{-13}$ & $3.0 \times 10^{-09}$ & $2.05 \times 10^{-1}$ & ${ }^{-} 5.0 \times 10^{-10}$ & $2.0 \times 10^{-09}$ & $2.0 \times 10^{-09}$ \\
\hline
\end{tabular}

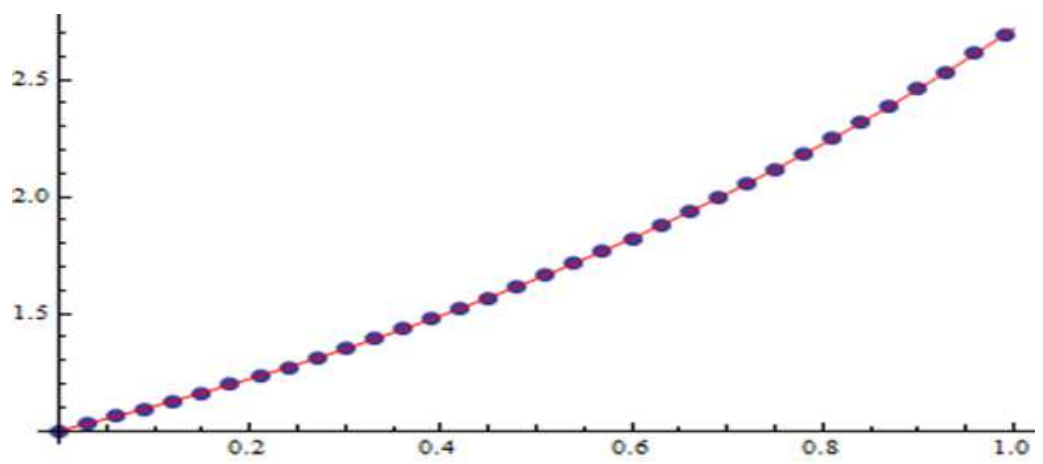

Figure 4: Dotted curve-sol: (MOHPM) and solid curve-sol: (Exact). 